\title{
Noise Analysis and Massive MIMO Modelling in VLC for 5G Networks using EKF with SCFDM
}

Sindhuja Rajesh ( $\square$ sindhujar@bmsce.ac.in )

BMSCE: BMS College of Engineering https://orcid.org/0000-0001-7334-462X

Arathi R Shankar

DBIT: Don Bosco Institute of Technology

\section{Research Article}

Keywords: 5G Networks, Allan Variance, Massive Multiple Input and Multiple Output, Orthogonal Frequency Division Multiplexing, Visible Light Communications, White Noise

Posted Date: February 20th, 2021

DOI: https://doi.org/10.21203/rs.3.rs-175826/v1

License: (1) This work is licensed under a Creative Commons Attribution 4.0 International License. Read Full License

Version of Record: A version of this preprint was published at Telecommunication Systems on August 22nd, 2021. See the published version at https://doi.org/10.1007/s11235-021-00824-8. 


\section{Noise Analysis and Massive MIMO Modelling in VLC for 5G Networks using EKF with SCFDM}

\author{
R. Sindhuja \\ Research Scholar, Dept. of ECE \\ BMS College of Engineering \\ Bangalore, India \\ sindhujar@bmsce.ac.in
}

\author{
Dr. Arathi R Shankar \\ $H O D$, Dept. of ECE \\ BMS College of Engineering \\ Bangalore, India \\ arathi.ece@bmsce.ac.in
}

\begin{abstract}
Visible Light Communications (VLC) is the type of communication, which processes high-speed data transmission using the visible Light Emitting Diodes (LED). The VLC acts as an important supplementary that is used to define the hotspots for heterogeneous networks and plays an important role for $5 \mathrm{G}$ networks in wireless communications. However, performance of visible light systems is affected by various noises and Allan variance is used to analyze such noises in 5G networks. The Massive Multiple-Input and MultipleOutput (M-MIMO) technique is used for noise modeling which utilizes the mitigation circuit to find whether the noise is white noise, shot noise, random walk noises or typical noises. The existing Kalman Filter approach failed to attain the required bandwidth and higher spectral efficiency. Therefore, to achieve high data rates, the spectral efficient technologies such as Single Carrier Frequency Division Multiplexing (SCFDM) is performed in the research. The Allan Variance is utilized for analyzing the time-series that extracts the noise features of the data and the major noise is verified and considered by the $M$ MIMO technique. The present research uses the Extended Kalman Filter (EKF) which determines the observation models and the state transition that does not need linear functions to define the states. The proposed SCFDM was constructed based on the VLC for $5 \mathrm{G}$ networks that analyzes in terms of Bit Error Rate (BER) and Signal to Noise Ratio (SNR). The proposed SCFDM obtains a high SNR of $14 \%$ for the channels with white LED option when compared to the existing methods.
\end{abstract}

Keywords-5G Networks, Allan Variance, Massive Multiple Input and Multiple Output, Orthogonal Frequency Division Multiplexing, Visible Light Communications, White Noise.

\section{INTRODUCTION}

In recent years, Optical Wireless Communication has received the huge attention of researchers due to its visible light spectrum in the wireless communication and also it offered a high bandwidth for high speed data transmission in wireless communication [1]. Additionally, it is possible to elaborate the usage of transfer data at an indoor optical wireless communication which carries the intrinsic characteristics of LED in VLC. To enhance the performance of the communication link, an optical MIMO communication is setup to achieve a high data rate transmission for mitigating shadow effects through indoor communication [2]. MIMO with OFDM is utilized for Optical wireless Communications that increase the data rate and spatial gain $[3,4]$. The OFDM and its modified versions modulate intensity that are directed to determine the intensity behaviour of VLC channels [5]. However, the intensity modulation and direct detection of VLC systems limit the direct application of MIMO and OFDM theory from wireless communications [6]. A dominant noise source is embedded on wireless optical channel which induces a shot noise in an indoor wireless optical channel which is being modelled with an Additive White Gaussian Noise (AWGN) [7]. An additive noise is added to the MIMO-VLC system such as constant stray light noise, shot noise, thermal noise, clipping noise, readout noise [8,9] to increase the Bit Error Rate (BER) of the system.

A multipath channel model for MIMO-VLC was developed in the existing methods to improve the channel capacity by performing the combination of M-MIMOOFDM. In order to analyze such noises, VLC system uses Allan variance for noise modelling and the mitigation circuit finds whether the noise is white noise or shot noise or random walk noises. Inside the mitigation circuit, digital signal processor is computed using Kalman filter. The SCFDM is proposed in VLC to decrease the complexity of the channel capacity and also it mitigates the noise using MIMO-OFDM. The time-correlated noises that existed in the VLC system are analyzed in the experimental studies.

The organization of this research paper is given as follows: Section II analyses the various research papers on noise detection in VLC systems. A detailed explanation of the proposed SCFDM is given in Section III. Section IV discusses the results in terms of quantitative and comparative analysis. The conclusion and future work of the proposed method is explained in Section V.

\section{LITERATURE REVIEW}

The various noises degrade the optical signal during transmission in the VLC system and this section reviews about distinct methodologies that were implemented to remove the noises in MIMO.

Li et al. [10] developed a multi-layered optical OFDM for high spectral efficiency in the visible light communication system. The noises such as AWGN, shot noise and thermal noise were analyzed in the developed system. The developed schemes were employed using Generalized Spatial Modulation with MIMO that performed coded modulation for the optical signals. However, the developed optical OFDM attained bandwidth utilization and it failed to achieve spectral efficiency at higher rate.

Farahneh et al. [11] developed an Adaptive optical OFDM scheme in the VLC system that executed by a Vehicular communication network for realistic environment. The developed model showed an improvement in data rates up to $50 \mathrm{Mbps}$ that achieved BER reduction under LOS and non- LOS conditions (high noise). The results obtained from the developed model showed the increase in data rate up to 50 Mbps with minimized BER under LOS and non-LOS conditions. However, the effect of various weather conditions such as rain, fog were not taken into consideration in this developed method.

Li et al. [12] analysed the spatial correlation for imaging MIMO operated underwater VLC. The channel capacity for 
the developed model consisted of upper bounds to solve power allocation problems. The added noise such as thermal noise and shot noise were removed from the developed model to improve the overall performance. The water injection method was utilized to achieve SNR at lower rates in under the same channel. However, increased SNR values of the system lead to a power allocation problem during water injection.

Dang et al. [13] developed a Novel Receiver for Asymmetrically Clipped Optical(ACO) OFDM in VLC. The developed ACO-OFDM are distorted due to the presence of subcarriers with the help of clipping noise. The novel receiver collected a minimum MSE which exploited the subcarriers to improve the BER performance. In general, the developed model showed highest complexity in the ACOOFDM which lowered the system performances.

Abdulkafi et al. [14] developed a $2 \times 2$ imaging MIMO system which was designed based on LED VLC that employed a space balanced coding for Positive Intrinsic Negative (PIN) array reception. In the developed model, noises such as AWGN and phase were added and modelled using PIN array reception. However, this developed model failed to achieve higher data and transmission for long distance system.

\section{PROPOSED SYSTEM}

An overview of the proposed VLC model is shown in figure1. The VLC system consists of SCFDM Transmitter, DC Bias Circuit, LED, Photo detector, Allan Variance, Noise Mitigation circuit, Kalman filter, SCFDM Receiver and random noises like white noise. The present research is efficient to analyze the noise during data transmission using Allan variance. The photoelectric sensor is used in this proposed SCFDM model to influence the VLC signal and other noises.

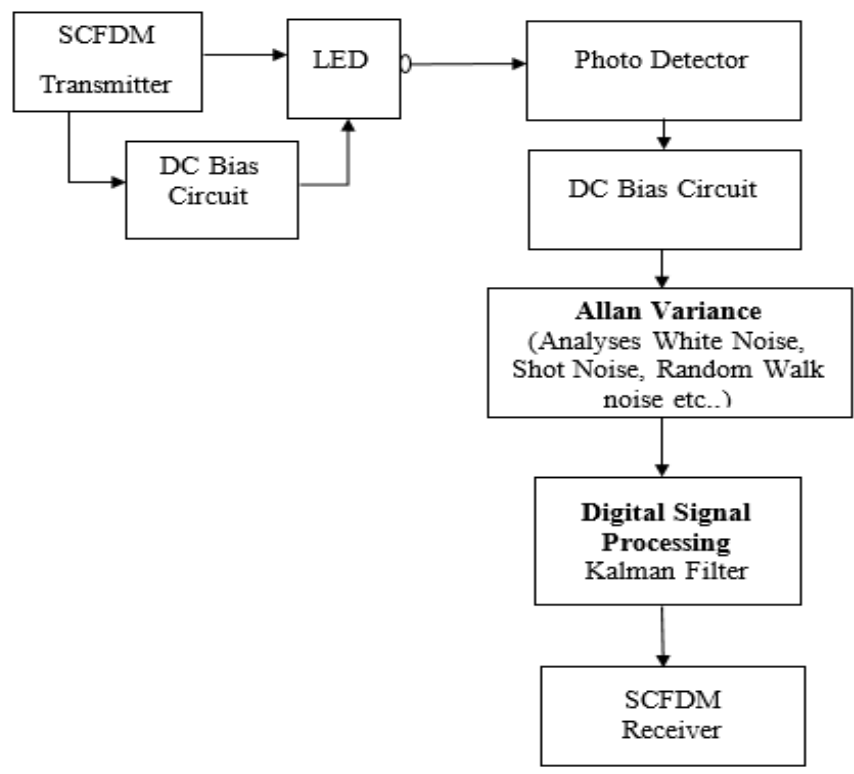

Fig. 1. Overview of the proposed method

\section{A. Allan Variance for Noise Measurement}

The noise measurement modules are used in the developed model for estimating Allan variance at every light source. The time-series analysis method uses Allan variance for extracting the noise and to remove the unwanted noise data. The RMSE values obtained by the randomly generated drift was based on the average time equation [15]. Allan Variance method is used to perform data instability with respect to distinct sampling time. The principles that are needed to be followed for noise measurement are given as follows:

$N_{S}$ represents the consecutive sampling data points,

$t_{0}$ is a sampling interval.

The $n$ consecutive sampling points at the first points are used for a cluster $\left(n<\frac{N_{S}}{2}\right)$ which calculates the mean value using Eq. (1).

$$
\overline{x_{k}}(n)=\frac{1}{n} \sum_{i=k}^{K+n-1} x_{i}
$$

Where $k=1,2, \ldots n$

The difference among the 2 adjacent data clusters are used for the next step by using the equation given in Eq. (2).

$$
\xi_{k+1, k}=\bar{x}_{k+1}(n)-\bar{x}_{k}(n)
$$

The Allan Variance consists of clusters and the length of clusters is represented in Eq. (3) and Eq. (4).

$$
\begin{aligned}
& \sigma^{2}(T)=\frac{1}{2}\left\langle\xi_{k+1, k}^{2}\right\rangle \\
& \sigma^{2}(T)=\frac{1}{2\left(N_{S}-2 n+1\right)} \sum_{k=1}^{N_{S}-2 n+1}\left(\bar{x}_{k+1}(n)-\bar{x}_{k}(n)\right)^{2}
\end{aligned}
$$

The continuously occurring random process is defined as the Power Spectral Density (PDS) which is represented as 
$S_{X}(f)$. The relationship between the Allan Variance and the PSD is expressed in Eq. (5).

$$
\sigma^{2}(T)=4 \int_{0}^{\infty} S_{X}(f) \frac{\sin ^{4}(\pi f T)}{(\pi f T)^{2}} d f
$$

The Allan Variance is used to detect Gaussian white noise that is calculated using Eq. (6).

$$
\sigma^{2}(T)=\frac{N^{2}}{T}
$$

Here, $N$ indicates the Gaussian white noise coefficient. The Allan Variance for the curve slope is $-\frac{1}{2}$. Where $N$ is represented in Eq. (7).

$$
N=\sigma(1)
$$

On the basis of Eq. (6), a linear log of $T$ is used, where $\delta$ is used as a percentage error. Allan Variance is estimated using $T$ as a specific cluster length expressed using the Eq. (8).

$$
\sigma(\delta)=\frac{1}{\sqrt{2\left(\frac{N_{S}}{n}-1\right)}}
$$

Allan Variance obtains an accurate high sampling time.

\section{B. Extended Kalman Filter (EKF) Algorithm for noise Mitigation}

The EKF is an improvised version of Kalman filter which linearizes the values of covariance and mean. The welldefined transition models are considered as a theory for nonlinear state estimation and for navigation of the systems. Therefore, major noise is particularly verified and considered for noise mitigation as the filters are applicable for real-time tracing. The mathematical expressions for the EKF to remove noise is done using Eq. (9) and Eq. (10).

$$
\begin{aligned}
& x_{k}=f\left(x_{k-1}, u_{k}\right)+w_{k} \\
& z_{k}=h\left(x_{k}\right)+v_{k}
\end{aligned}
$$

Here, $w_{k}$ and $v_{k}$ are the process and observation noises and $u_{k}$ is the control vector.

$f$ is the function used to calculate the present state based on the previous estimation of function.

$h$ is the function is used to compute the predicted measurement from predicted states. The Kalman filter and particle filter performs the operation at the noise position to make the noise well fined. The present research uses EKF that performs dynamic test, thereby compensating for the difficulty of Kalman filter that works for non-linear systems. The EKF is transformed using Taylor series that obtains an approximation linearization model [16]. The co-variance matrix $R$ is formed based on the signal noise for Allan
Variance utilization. The working process of EKF is explained as follows,

\section{Input:}

$\left\{s_{k-1}, P_{k-1}\right\}$ is the state for the covariance at time phase $k-1$

$m_{k}$ time step measurement at time $k$

\section{Output:}

$\left\{s_{k}, P_{k}\right\}$ state for the covariance at time phase $k$

\section{Process:}

1. The initial state is set at $k$ that is based on the start point location to provide the values using ALSQ algorithm. The initial covariance is set as $P_{k}$ with non-zero matrix $R$ and it also consists of noise element $\sigma_{\text {noise }}$.

2. Linearization process is performed using the Eq. (11).

$$
\begin{gathered}
A=\frac{\partial f_{S 1}}{\partial X}=\left[\begin{array}{ccc}
\frac{\partial f_{s 1}}{\partial X} & \cdots & \frac{\partial f_{s 1}}{\partial X} \\
\mathrm{M} & \mathrm{O} & \mathrm{M} \\
\frac{\partial f_{s n}}{\partial X_{1}} & \cdots & \frac{\partial f_{s n}}{\partial X_{n}}
\end{array}\right] \\
H=\frac{\partial h_{0}}{\partial X}=\left[\begin{array}{lll}
\frac{\partial h_{o 1}}{\partial x_{1}} & \mathrm{~L} & \frac{\partial h_{o 1}}{\partial x_{n}} \\
\mathrm{M} & \mathrm{O} & \mathrm{M} \\
\frac{\partial h_{o n}}{\partial x_{1}} & \mathrm{~L} & \frac{\partial h_{o n}}{\partial x_{n}}
\end{array}\right]
\end{gathered}
$$

3. For $i=1: 1:$ step $_{\text {num }}$

4. Predict the state vector $S_{\bar{k}}=A s_{k-1}$;

5. Predict the state covariance matrix $P_{\bar{k}}=A P_{k-1} A^{T}+Q$

6. Compute the Kalman gain $K_{k}=P_{k}^{-} H^{T}\left(H P_{k}^{-} H^{T}+R\right)$

7. Update the state vector $S_{k}=S_{k}^{-}+K_{k}\left(m_{k}-H s_{k}^{-}\right)$

8. Update covariance matrix $P_{k}=\left(I-K_{k} H\right) P_{k}^{-}$

9. End For

10. Final.

\section{Single Channel Frequency Division Multiplexing Model}

The block diagram of the SCFDM system in VLC is represented in figure 2 and figure 3 . Figure 2 shows the diagram of the SCFDM system in VLC (Transmitter) and Figure 3: shows the diagram of the SCFDM system in VLC (Receiver). 


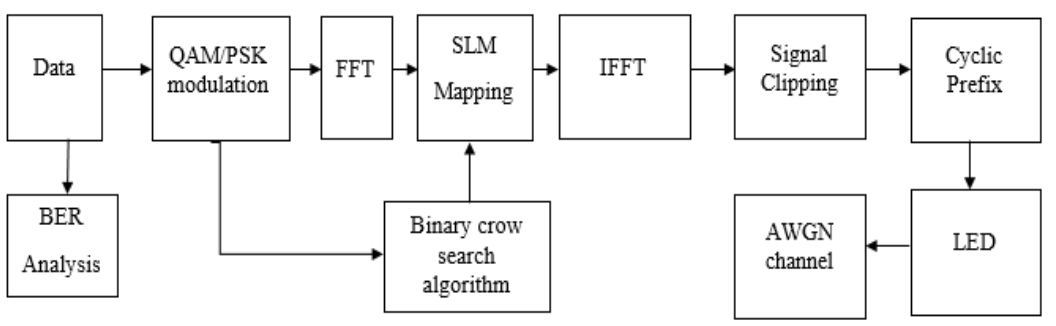

Fig. 2. The diagram of the SCFDM system in VLC (Transmitter)

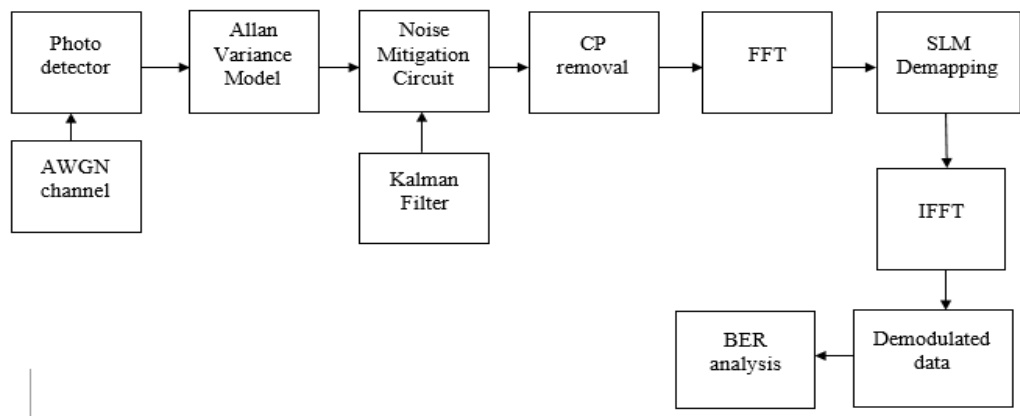

Fig. 3. The diagram of the SCFDM system in VLC (Receiver)

The proposed SCFDM-VLC system is used for mapping and it generated the sequence into 64 sub-carriers using PSK and QAM modulations. The PSK is used as a common digital modulation where the data transmission is performed to alter the phase of signal reference frequency. The QAM is used for both amplitude components and phases that consider the reference frequency signal. The QAM is utilized for both amplitude and phase components that deliver to form modulation at high level spectrum efficiency. The Selective Mapping (SLM) technique is applied for PAPR reduction that chooses the phase rotation factors effectively $[17,18]$.

$$
Y_{\text {equalized }}=\frac{Y}{C}=X+\frac{n}{C}
$$

Here,

$X$ is transmitted signal,

$n$ is noise term,

$Y$ is the frequency of received signal,

$C$ is frequency response for the channel.

The frequency response of the channel is defined using the training sequences. The equalizer coefficients reduce MMSE criterion where ISI and additive noise affect the system. The equalizer coefficients are minimized using Eq. (13).

$$
C_{M M S E}=\frac{\boldsymbol{H}}{|\boldsymbol{H}|^{2}+\sigma_{w}^{2} / \sigma_{X}^{2}}
$$

Where, $\sigma_{w}^{2}$ is variance obtained using additive noise, $H$ is channel matrix for the transmitter and receiver, and $\sigma_{X}^{2}$ are data symbols that are transmitted.

\section{White LED characteristics}

The SCFDM-VLC system used to transmit the sub-carrier signals which makes the small bandwidth difficult for few concerns such as demodulation errors, Inter-Symbol Interference (ISI), and orthogonality between the subcarriers. In order to overcome such consequences, the Cyclic Prefix (CP) is used for the channel that estimates the time length based on the delay. Figures 2 and 3 indicates the graphical depiction of proposed SCFDM based VLC system. The zero forcing operation is mathematically denoted in Eq. (12).

In the present research, white LED is used as a lighting device with the property of robustness, high lifetime, energy efficiency and decent temporal stability. The White LED consists of 2 features such as luminous intensity and transmitted optical power. The luminous intensity is denoted based on the white LED brightness which is calculated using the Eq. (14).

$$
I=\frac{d \phi}{d \delta}
$$

Where, $\phi$ is luminous flux, luminous flux $\phi_{e}$ is denoted using Eq. (15).

$$
\phi=K_{m} \int_{380}^{780} v(\lambda) \phi_{e}(\lambda) d \lambda
$$

Where, $K_{m}$ is the maximum visibility having the range of $680 \mathrm{~lm} / \mathrm{W}$ at $\lambda=555 \mathrm{~nm}, v(\lambda)$ is known called as luminosity curve. The energy flux integral $\phi_{e}$ at the transmitted optical power $P_{t}$ for all the direction is expressed using the Eq. (16).

$$
P_{t}=\int_{\wedge}^{\wedge} \min \int_{0}^{2 \pi} \phi_{e} d \phi d \lambda
$$

Where, $\wedge$ max and $\wedge$ min are sensitivity curve for the photodiode. 
At the angle $\phi$ luminous intensity is expressed mathematically using the Eq. (17).

$$
I(\phi)=I(0) \cos ^{m}(\phi)
$$

The horizontal illuminance $E_{\text {hor }}$ at a point $(x, y)$ is stated in Eq. (18).

$$
E_{\text {hor }}=\frac{I(0) \cos ^{m}(\phi)}{d^{2}} \times \cos (\psi)
$$

By using the Eq. (18), $\psi$ is called as a angle of incidence

The $I(0)$ is the Luminous intensity

$\phi$ is the angle of irradiance

$d$ is known as the distance between the detector surface of an LED

$m$ is known as the lambertain emission expressed mathematically using the Eq. (19). The parameter settings of white LEDs used in this research are shown in the table 1 as follows:

$$
m=\ln 2 / \ln \left(\cos \phi_{\frac{1}{2}}\right), \phi_{\frac{1}{2}}=60 \operatorname{deg} \text { ree }
$$

TABLE I. PARAMETER SETTINGS

\begin{tabular}{|c|c|}
\hline Number of LEDs & {$[64 \times 64]$} \\
\hline LEDs turn on voltage & $3 \mathrm{~V}$ \\
\hline bandwidth of LEDs & $10 \mathrm{MHz}$ \\
\hline LEDs turn off voltage & $4 \mathrm{~V}$ \\
\hline
\end{tabular}

\section{E. PAPR reduction using selective mapping}

Initially, the SCFDM -VLC is divided into equal spaced signals to achieve lesser signal distortions for wireless communication system that achieves data transmission at higher rate. The input frequency domain sequences are denoted mathematically using the Eq. (20)

$$
x=\left[x_{0}, x_{1}, \ldots, x_{n-1}\right]
$$

Where, $x$ is an input data, $n$ is data dimension.

The signals are modulated from the input for every time domain set which is computed using the SCFDM-VLC that encoded the digital data for each and every carrier frequency. The processed IFFT signals are expressed mathematically using the Eq. (21).

$$
X_{N}=\frac{1}{\sqrt{n}} \sum_{k=0}^{N-1} x_{N} W_{n}^{k N}
$$

Where, $k N$ is value for the single carrier frequency

$X_{N}$ is the signal that is processed using IFFT.

The high power generation is represented based on the calculation of the ratio between the average and maximum power that is expressed mathematically using the Eq. (22).

$$
P A P R=10 \log _{10} \frac{\max \left\{\left|X_{N}\right|^{2}\right\}}{E\left\{\left|S_{N}\right|^{2}\right\}}
$$

Where, $E\left\{\left|S_{N}\right|^{2}\right\}$ is known as the average power generated for $X_{N}$.

The IFFT output applied for SLM is calculated using the Eq. (23).

$$
X_{t}=\frac{1}{\sqrt{L n}} \sum_{k=0}^{L N-1} \bar{x}_{N} W_{n}^{k L N}, 0 \leq N \leq L N-1
$$

Where, $\bar{x}_{N}$ known modified input signal at $L=5$

\section{F. Sub-block partition optimization using BCS algorithm}

The BCS algorithm is an optimization based algorithm that mimics the crow's behaviour. For the optimization algorithms, the crows position $i$ is specified using the mathematical Eq. (24).

$$
x^{i, t}=\left[x_{1}^{i, t}, x_{2}^{i, t}, x_{3}^{i, t}, \ldots x_{d}^{i, t}\right]
$$

From the Eq. (24), $i$ is the value which ranges from 1 to $N, t$ ranges from 1 to $t \max$.

Similarly, the crows memorize the best locations or positions where it will get best food source which is denoted in Eq. (25). The BCS algorithm is used to update the position of crows in two dissimilar ways as mentioned in Eq. 25.

$$
m^{i, t}=\left[m_{1}^{i, t}, m_{2}^{i, t}, m_{3}^{i, t}, \ldots m_{d}^{i, t}\right]
$$

Case 1: Crow $j$ does not know the position of crow $i$. Subsequently, crow $i$ approaches crow $j$ and then the new location of crow $i$ is updated as shown in the Eq. (26).

$$
x_{1}^{i, t+1}=x^{i, t}+r_{j} \times f l^{i, t} \times\left(m^{j, t}-x^{i, t}\right)
$$

Where, $f l^{i, t}$ indicated as flight length of crow, $i$ is the iteration at $t$, and $r_{j}$ is represented as random number that ranges between $[0,1]$.

Case 2: Crow $j$ knows crow $i$ position. When the crow $j$ distracts crow $i$ by moving elsewhere in the search space to protect its hiding place, a novel position of the crow $i$ is randomly updated as expressed in the Eq. (27).

$x_{1}^{i, t+1}=\left\{\begin{array}{c}x^{i, t}+r_{j} \times f l^{i, t} \times\left(m^{j, t}-x^{i, t}\right) r_{j} \geq A P^{j, t} \\ \text { Random posirion }\end{array}\right.$

otherwise

Where, $A P^{i, t}$ is known as the Awareness Probability (AP) of crow at the $j$ generation

\subsubsection{Pseudo code of BCS algorithm}

Initialize, the crow's position $N$ and the memory of every crow

While maximum generation is not researched do

For $i=1: N$ 
Randomly select one crow, and define the awareness probability.

$$
\begin{aligned}
& \text { If } r_{j}>A P^{j, t} \\
& x_{1}^{i, t+1}=x^{i, t}+r_{j} \times f l^{i, t} \times\left(m^{j, t}-x^{i, t}\right)
\end{aligned}
$$

\section{Else}

$x^{i, t+1}$ is denoted as random position of the search space.

\section{End if}

\section{End for}

Verify the feasibility of crow's new position.

Calculate the crow's new position.

Update the crow's memory.

\section{End while}

End

\section{RESULTS}

TABLE II. SIMULATION PARAMETERS

\begin{tabular}{|c|c|c|}
\hline Parameter & Notation & Value \\
\hline LED modulation bandwidth & $W_{L E D}$ & $\mathbf{5 0 ~ M H z}$ \\
\hline Semi-angle at half power & $\theta_{\frac{1}{2}}$ & $60^{\circ}$ \\
\hline FOV & $\varphi_{F O V}$ & $80^{\circ}$ \\
\hline Area of Photo Detector & $A_{R}$ & $0.1 \mathrm{~cm}^{2}$ \\
\hline Reflectivity of wall & - & 0.8 \\
\hline Area of reflecting elements & $\Delta A$ & $0.01 \mathrm{~m}^{2}$ \\
\hline
\end{tabular}

The results for the proposed SCFDM is designed based on the VLC system which are simulated using MATLAB (2018a). The results obtained for the performance metrics are compared against the modulation techniques which includes
QAM and PSK. These are evaluated in terms of MMSE and Zero forcing that evaluates the efficiency of the proposed method. The main simulation parameters are presented Table 2.

\section{A. Performance measure}

In SCFDM, BER is evaluated using received bits along with AWGN. The AWGN is altered by performing distortion, synchronization errors, interference and noise. The SNR defines the ratio of Signal Power to the Noise Power (dB) and is expressed in Eq. (28).

$$
S N R=\frac{P_{\text {Signal }}}{P_{\text {Noise }}}
$$

\section{B. Quantitative analysis}

The parameters used for simulation environment are shown in the table 2. The LEDs receive a signal and are simulated by using the Photo Detector Channel model having the random Gaussian noise, whose noise is having the variance which is set by using the Allan Variance which obtains the results for the field tests. The results which are obtained for positioning before and after the denoising are measured as shown in the table 3 .

In the proposed systems, the $16 \times 16$ elements evaluated in terms of SNR is compared to the existing system and simulations results are shown in Figures 4 and 5. However, the values are mainly affected by the geometry distribution between the receiver and LED. The proposed denoised process improves the SNR rate of the VLC system when compared with the Existing Conventional SCFDMA. The SNR analysis of the proposed system for 16X16 elements is

\begin{tabular}{|c|c|c|c|c|c|}
\hline \multicolumn{6}{|c|}{ SNR estimation for $16 \times 16$ elements } \\
\hline Length of the room (x-axis) & 2 & 1 & 0 & -1 & -2 \\
\hline Width of the room (y-axis) & 2 & 1 & 0 & -1 & -2 \\
\hline SNR $(\mathrm{dB}) \mathrm{z}$ axis & 0 & 10 & 20 & 30 & 40 \\
\hline Existing Conventional SCFDMA (SNR) [15] & 9.32 & 15.25 & 23.109 & 29.23 & 35.78 \\
\hline Proposed SCFDMA (SNR) & 11.35 & 19.64 & 28.424 & 34.864 & 40.6619 \\
\hline
\end{tabular}
given in Table 3.

TABLE III. SNR ANALYSIS OF PROPOSED SYSTEM FOR 16X16 ELEMENTS

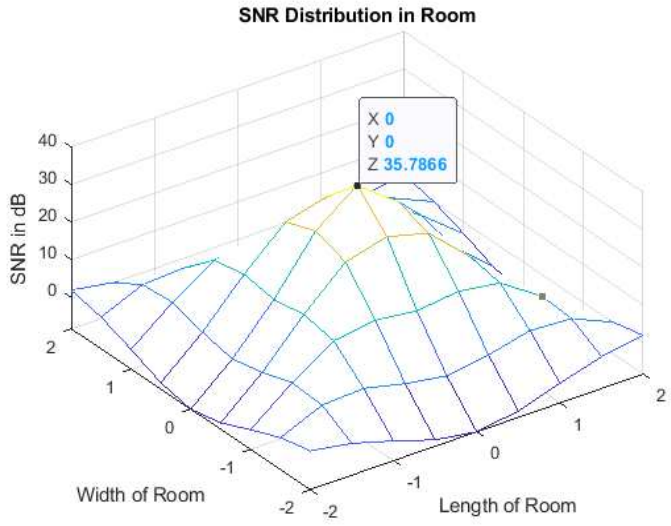

Fig. 4. SNR analysis of existing system for $16 \mathrm{X} 16$ elements

Correspondingly, table 4 analyzed the performance of the proposed system in light of SNR for 32 X 32 elements. Table 4 consists of SNR values obtained for both existing and

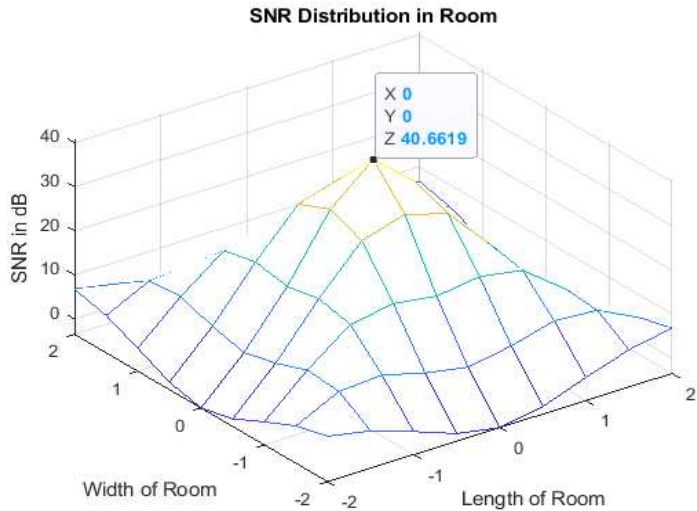

Fig. 5. SNR analysis of proposed system for $16 \mathrm{X} 16$ elements

proposed method that are plotted in the graph as shown in the figure 6. Similarly, table 4 consists of SNR values that are plotted in the graph for the existing Conventional SCFDMA 
method and the proposed SCFDMA which is represented as shown in figure 7.

TABLE IV. SNR ANALYSIS OF PROPOSED SYSTEM FOR 32X32 ELEMENTS

\begin{tabular}{|c|c|c|c|c|c|}
\hline \multicolumn{6}{|c|}{ SNR estimation for $32 \times 32$ elements } \\
\hline Length of the room (x-axis) & 2 & 1 & 0 & -1 & -2 \\
\hline Width of the room (y-axis) & 2 & 1 & 0 & -1 & -2 \\
\hline SNR $(\mathrm{dB}) \mathrm{z}$ axis & 0 & 10 & 20 & 30 & 40 \\
\hline Existing Conventional SCFDMA (SNR) & 11.34 & 18.24 & 26.24 & 32.54 & 40.66 \\
\hline Proposed SCFDMA (SNR) & 13.56 & 20.42 & 28.453 & 39.43 & 43.12 \\
\hline
\end{tabular}

Table 5 shows the tabulation for BER vs SNR for hybrid Kalman Filter and SCFDMA for $16 \times 16$ elements. Figure 8 shows the BER vs SNR for hybrid Kalman Filter and SCFDMA for $16 \times 16$ elements.

TABLE V. BER VS SNR FOR HYBRID KALMAN FILTER AND SCFDMA FOR $16 \times 16$ ELEMENTS

\begin{tabular}{|c|c|c|c|c|c|c|c|c|c|c|c|c|c|}
\hline $16 \times 16(\mathrm{SNR}$ in $\mathrm{dB})$ & -30 & -25 & -20 & -15 & -10 & -5 & $\mathbf{0}$ & 5 & 10 & 15 & 20 & 25 & 30 \\
\hline Hybrid(SNR) & 0.2482 & 0.240 & 0.229 & 0.204 & 0.166 & 0.113 & 0.058 & 0.024 & 0.009 & 0.004 & 0.002 & 0.00088 & 0.00079 \\
\hline Conv(SNR) & 0.492 & 0.481 & 0.458 & 0.406 & 0.334 & 0.230 & 0.119 & 0.051 & 0.020 & 0.007 & 0.004 & 0.0017 & 0.0014 \\
\hline
\end{tabular}

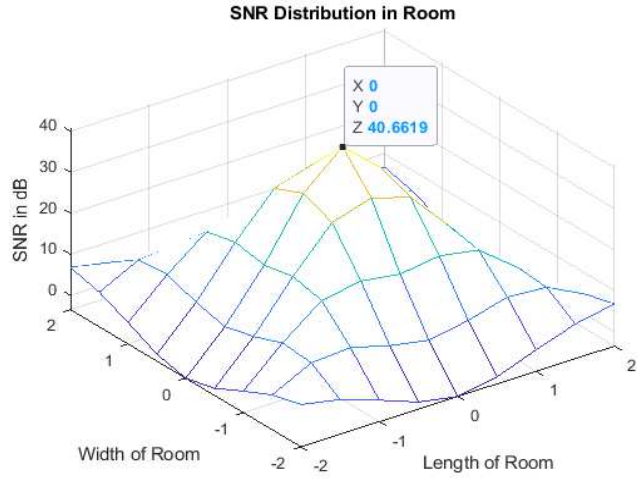

Fig. 6. SNR analysis of existing system for 32X32 elements

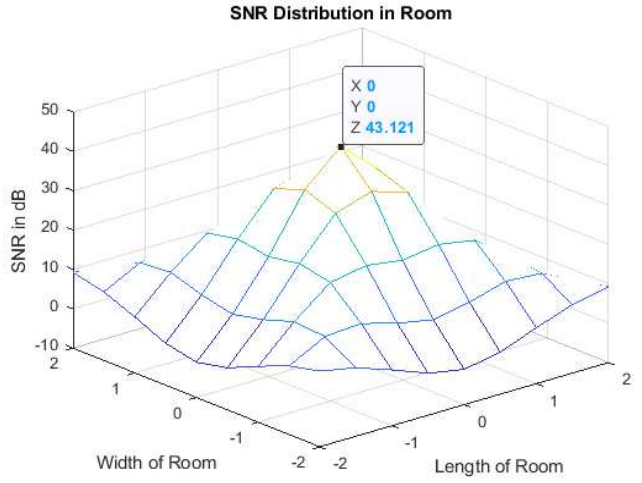

Fig. 7. SNR analysis of proposed system for $32 X 32$ elements

Table 6 shows the values for BER and SNR using the hybrid Kalman Filter for $32 \times 32$ elements. The values obtained from the table 6 is graphically represented in figure 9 for hybrid Kalman Filter and SCFDMA.

TABLE VI. BER VS SNR FOR HYBRID KALMAN FILTER AND SCFDMA FOR $32 \times 32$ ELEMENTS

\begin{tabular}{|c|c|c|c|c|c|c|c|c|c|c|c|c|c|}
\hline $32 \times 32 \mathrm{SNR}$ in $\mathbf{~ d B}$ & -30 & -25 & -20 & -15 & -10 & -5 & 0 & 5 & 10 & 15 & 20 & 25 & 30 \\
\hline Hybrid (SNR) & 0.2476 & 0.2407 & 0.2294 & 0.2041 & 0.1660 & 0.1138 & 0.0588 & 0.0236 & 0.0097 & 0.00373 & 0.00247 & 0.00152 & 0.00093 \\
\hline Conv(SNR) & 0.4928 & 0.4818 & 0.4589 & 0.4063 & 0.3344 & 0.2300 & 0.1195 & 0.05103 & 0.0202 & 0.00824 & 0.00432 & 0.00215 & 0.00092 \\
\hline
\end{tabular}

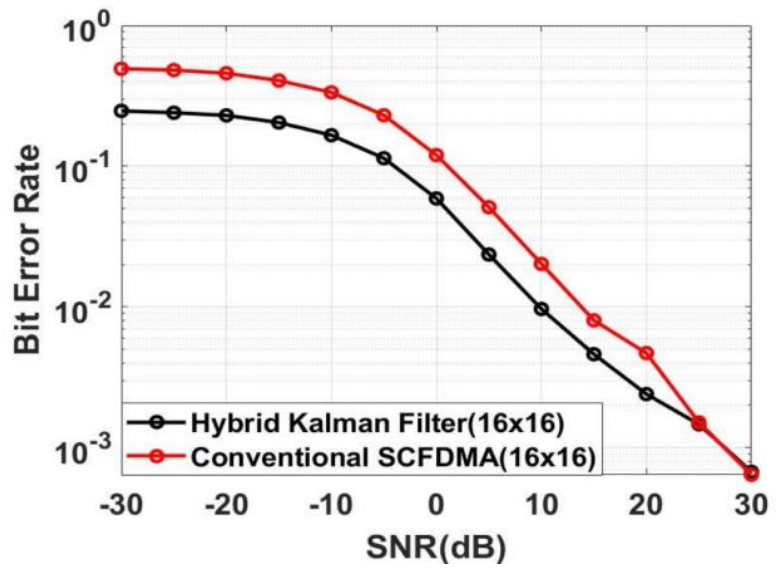

Fig. 8. BER vs SNR for hybrid Kalman Filter and SCFDMA for $16 \times 16$ elements

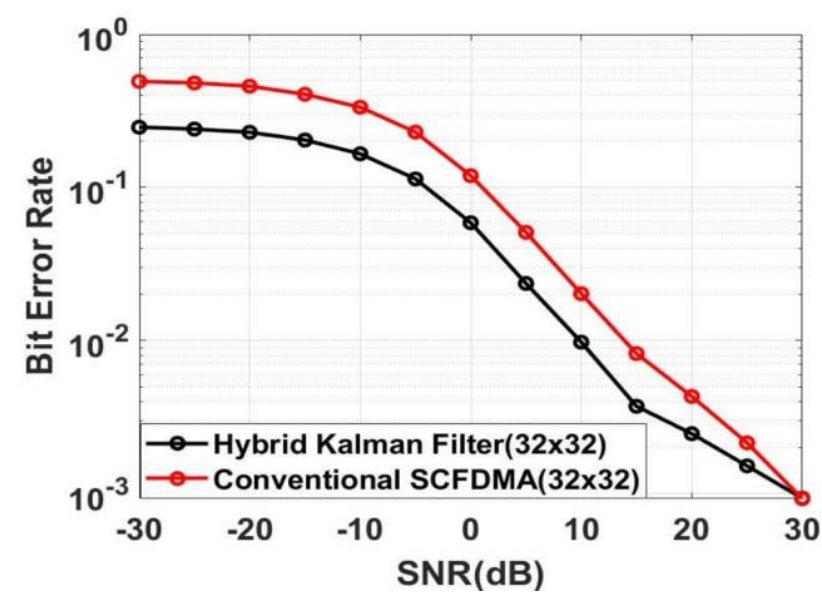

Fig. 9. BER vs SNR for hybrid Kalman Filter and SCFDMA for $32 \times 32$ elements 


\section{Comparitive Analysis}

Table 7 shows the performance executed by the existing ALSQ and EKF. The results obtained by the developed model out performed using an average filter and Allan Variance. The improvement of the SNR in existing method are that, Adaptive Least Squares (ALSQ), EKF [19] showed
$19 \%$ improvement, Modelling in Visible Light Communication Using Allan Variance [15] showed 9\% improvement whereas, the proposed method showed $14 \%$ improvement in SNR. Table 7 showed improved BER performance for the $6 \mathrm{~m} \times 6 \mathrm{~m} \times 4 \mathrm{~m}$ theoretical room dimension.

TABLE VII. SNR COMPARISON BY ANALYZING THE PROPOSED SYSTEM AND THE EXISTING SYSTEM.

\begin{tabular}{|c|c|c|}
\hline \multicolumn{2}{|c|}{ SNR comparison } \\
\hline Methodologies & Room Dimension & Improvement of SNR \\
\hline Zhuang, Y. et al [19] & $5 \mathrm{~m} \times 5 \mathrm{~m} \times 2.843 \mathrm{~m}$ & $19 \%$ \\
\hline Hua, L et al [15] & $3 \mathrm{~m} \times 3 \mathrm{~m} \times 0.85 \mathrm{~m}$ & $9 \%$ \\
\hline Proposed & $6 \mathrm{~m} \times 6 \mathrm{~m} \times 4 \mathrm{~m}$. & $14 \%$ \\
\hline
\end{tabular}

\section{CONCLUSION}

In the research, the proposed EKF-SCFDMA model was used for noise measurement of visible light system based on the Allan Variance. A realistic VLC for $5 \mathrm{G}$ networks was built and field tests were developed in the study that measured and evaluated the VLC noise in M-MIMO using the proposed scheme. The obtained results showed that two types of noises namely white noise and short cluster time noise were dominant in the system. The results for simulation showed that Allan Variance obtained better performance when compared with existing methods in VLC for $5 \mathrm{G}$ networks. According to the field static tests, the proposed VLC system obtained SNR of $43.12 \mathrm{~dB}$ in $32 \times 32$ elements with an improvement of $14 \%$ with the existing method. The proposed SCFDMA showed that the EKF with Allan Variance removed the noise components to improve the performance in terms of SNR ratio. The results were transparent and showed that Allan Variance technique was efficient for measuring noises in VLC 5G networks. The simulation field tests showed improved SNR for room dimension of $6 \mathrm{~m} \times 6 \mathrm{~m} \times 4 \mathrm{~m}$. In the future, the performance evaluation can be improved for large scale environment.

\section{REFERENCES}

[1] A. A. Abdulkafi, S. M. Hardan, O. Bayat, and O. N. Ucan, "Multilayered optical OFDM for high spectral efficiency in visible light communication system," Photonic Network Communications, vol. 38, no. 3, pp. 299-313, December 2019.

[2] J. Tan, K. Yang, and M. Xia, "Adaptive equalization for high speed optical MIMO wireless communications using white LED," Frontiers of Optoelectronics in China, vol. 4, no. 4, pp. 454-461, December 2011.

[3] L. Wu, Z. Zhang, and H. Liu, "MIMO-OFDM visible light communications system with low complexity," IEEE international conference on communications, pp. 3933-3937, June 2019.

[4] P.P. Banik, R. Saha, and K.D. Kim, "Regression analysis for LED color detection of visual-MIMO system," Opt. Commun., vol. 413, pp. 121-130, April 2018.

[5] A. Yesilkaya, E. Basar, F. Miramirkhani, E. Panayirci, M. Uysal, and H. Haas, "Optical MIMO-OFDM with generalized LED index modulation," IEEE Trans. Commun., vol. 65, no. 8, pp. 3429-3441, May 2017.

[6] Y. Hong, T. Wu, and L. K. Chen, "On the performance of adaptive MIMO-OFDM indoor visible light communications," IEEE Photonics Technol. Lett., vol. 28, no. 8, pp. 907-910, January 2016.
[7] Z. Ghassemlooy, C. Ma, and S. Guo, "PAPR reduction scheme for ACO-OFDM based visible light communication systems," Opt. Commun., vol. 383, pp. 75-80, January 2017.

[8] R. Wu, Y. Guo, J. Liu, and P. Liu, "Modeling and analysis of spatial inter-symbol interference for MIMO image sensors based visible light communication," In 2017 ITU Kaleidoscope: Challenges for a DataDriven Society (ITU K), pp. 1-7,November 2017.

[9] K. Jia, and L. Hao, "Modeling of Multipath Channel and Performance Analysis of MIMO-DCO-OFDM System in Visible Light Communications," Chinese Journal of Electronics, vol. 28, no. 3, pp. 630-639, May 2019.

[10] J. Li, Y. Xu, J. Shi, Y. Wang, X. Ji, H. Ou, and N. Chi, “A $2 \times 2$ imaging MIMO system based on LED Visible Light Communications employing space balanced coding and integrated PIN array reception," Opt. Commun., vol. 367, pp. 214-218, May 2016.

[11] H. Farahneh, F. Hussain, and X. Fernando, "Performance analysis of adaptive OFDM modulation scheme in VLC vehicular communication network in realistic noise environment," EURASIP Journal on Wireless Communications and Networking, vol. 2018, no. 1, pp. 243, December 2018.

[12] Y. Li, H. Qiu, X. Chen, J. Fu, M. Musa, and X. Li, "Spatial correlation analysis of imaging MIMO for underwater visible light communication," Opt. Commun., vol. 443, pp. 221-229, July 2019.

[13] J. Dang, Z. Zhang, and L. Wu, "A novel receiver for ACO-OFDM in visible light communication,” IEEE Commun. Lett., vol. 17, no. 12, pp. 2320-2323, November 2013.

[14] A. A. Abdulkafi, S. M. Hardan, O. Bayat, and O. N. Ucan, "Multilayered optical OFDM for high spectral efficiency in visible light communication system," Photonic Network Communications, vol. 38, no. 3, pp. 299-313, December 2019.

[15] L. Hua, Y. Zhuang, L. Qi, J. Yang, and L. Shi, "Noise analysis and modeling in visible light communication using Allan variance," IEEE Access, vol. 6, pp. 74320-74327, December 2018.

[16] Y. Zhuang, L. Hua, Q. Wang, Y. Cao, Z. Gao, L. Qi, J. Yang, and J. Thompson, "Visible Light Positioning and Navigation Using Noise Measurement and Mitigation," IEEE Transactions on Vehicular Technology, vol. 68, no. 11, pp. 11094-11106, September 2019.

[17] K. Jia, and L. Hao, "Modeling of Multipath Channel and Performance Analysis of MIMO-DCO-OFDM System in Visible Light Communications," Chinese Journal of Electronics, vol. 28, no. 3, pp. 630-639, May 2019.

[18] S. Dimitrov, S. Sinanovic, and H. Haas, "Clipping noise in OFDMbased optical wireless communication systems," IEEE Trans. Commun., vol. 60, no. 4, pp. 1072-1081, March 2012.

[19] Y. Zhuang, L. Hua, Q. Wang, Y. Cao, Z. Gao, L. Qi, J. Yang, and J. Thompson, "Visible Light Positioning and Navigation Using Noise Measurement and Mitigation," IEEE Trans. Veh. Technol., vol. 68, no. 11, pp. 11094-11106, September 2019. 
Figures

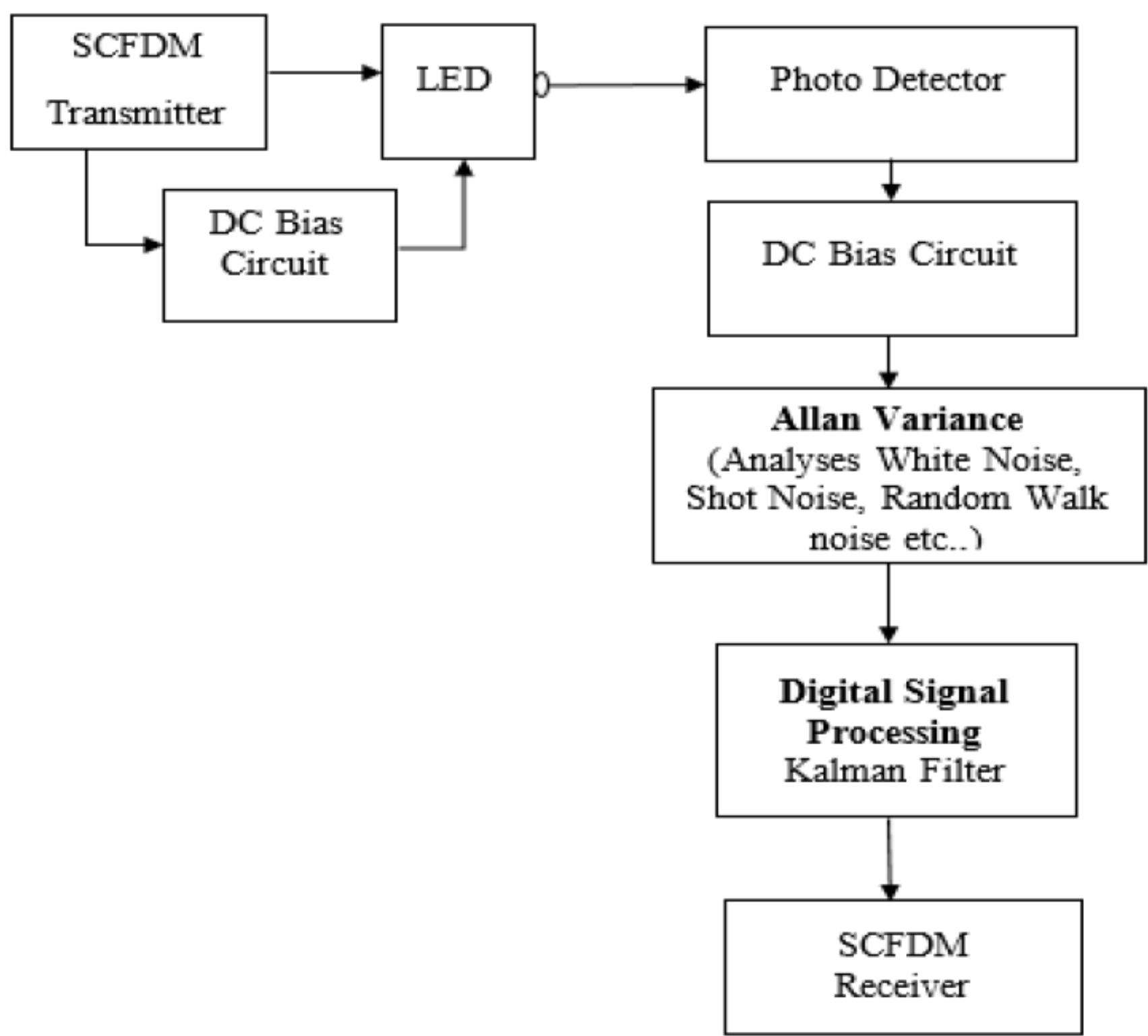

Figure 1

Overview of the proposed method 


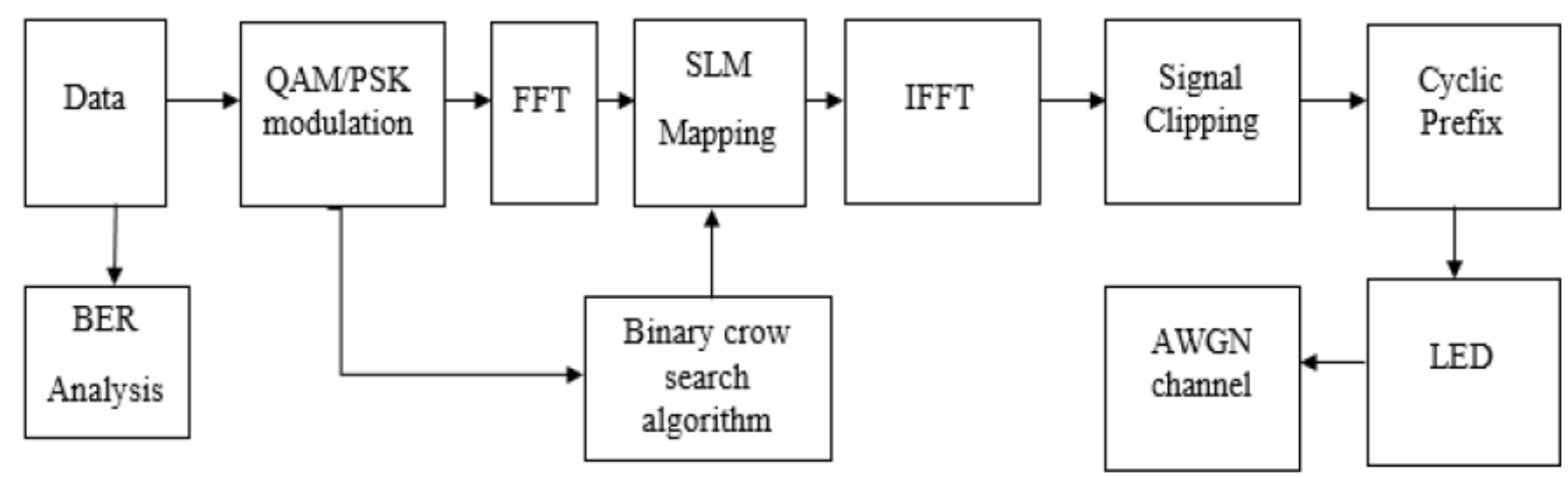

Figure 2

The diagram of the SCFDM system in VLC (Transmitter)

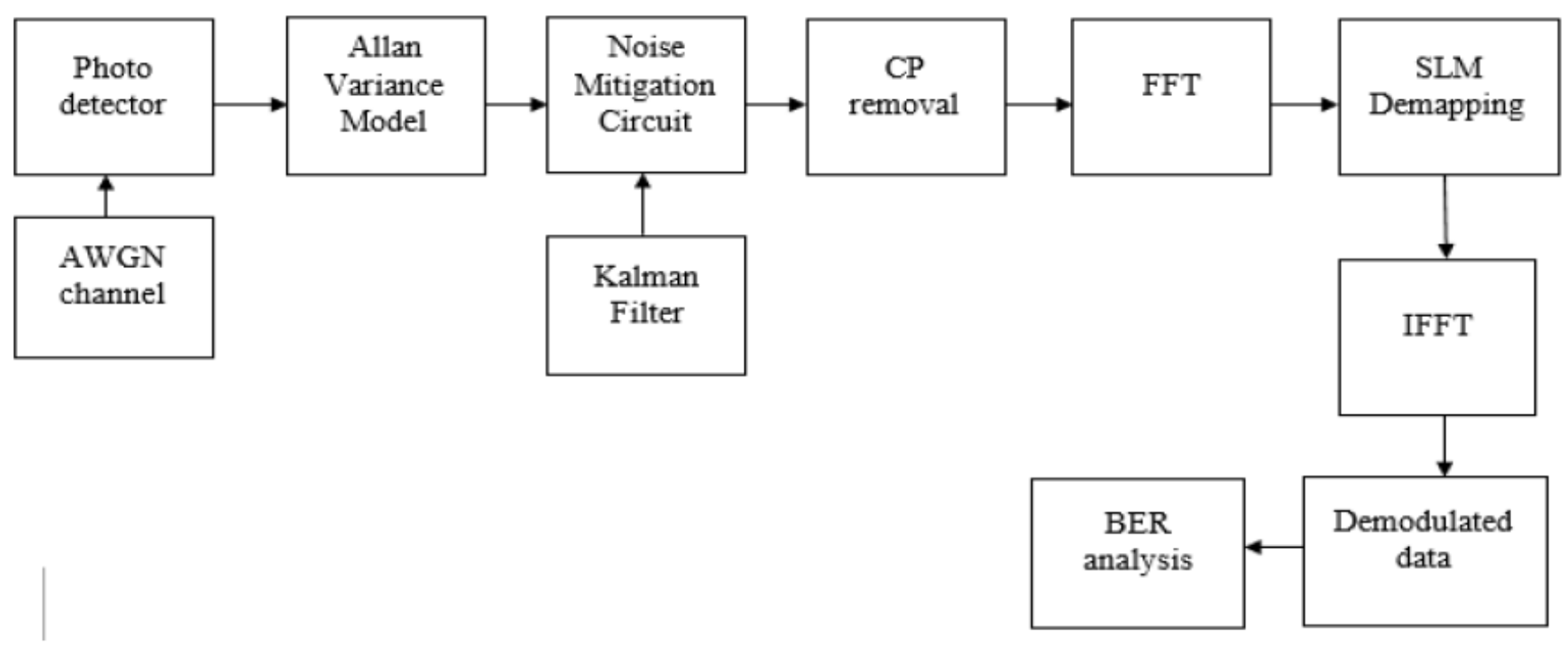

Figure 3

The diagram of the SCFDM system in VLC (Receiver) 


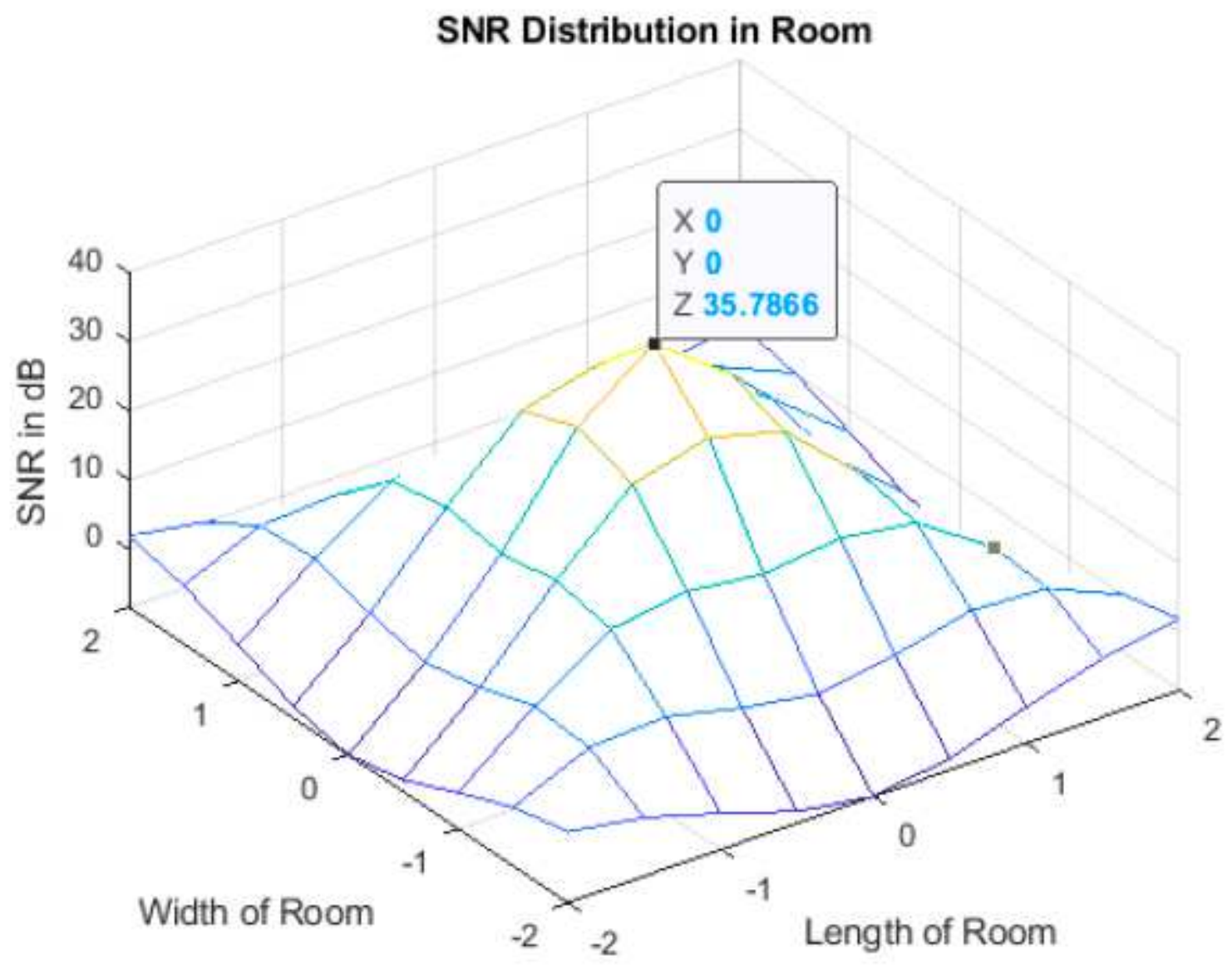

Figure 4

SNR analysis of existing system for $16 \times 16$ elements 


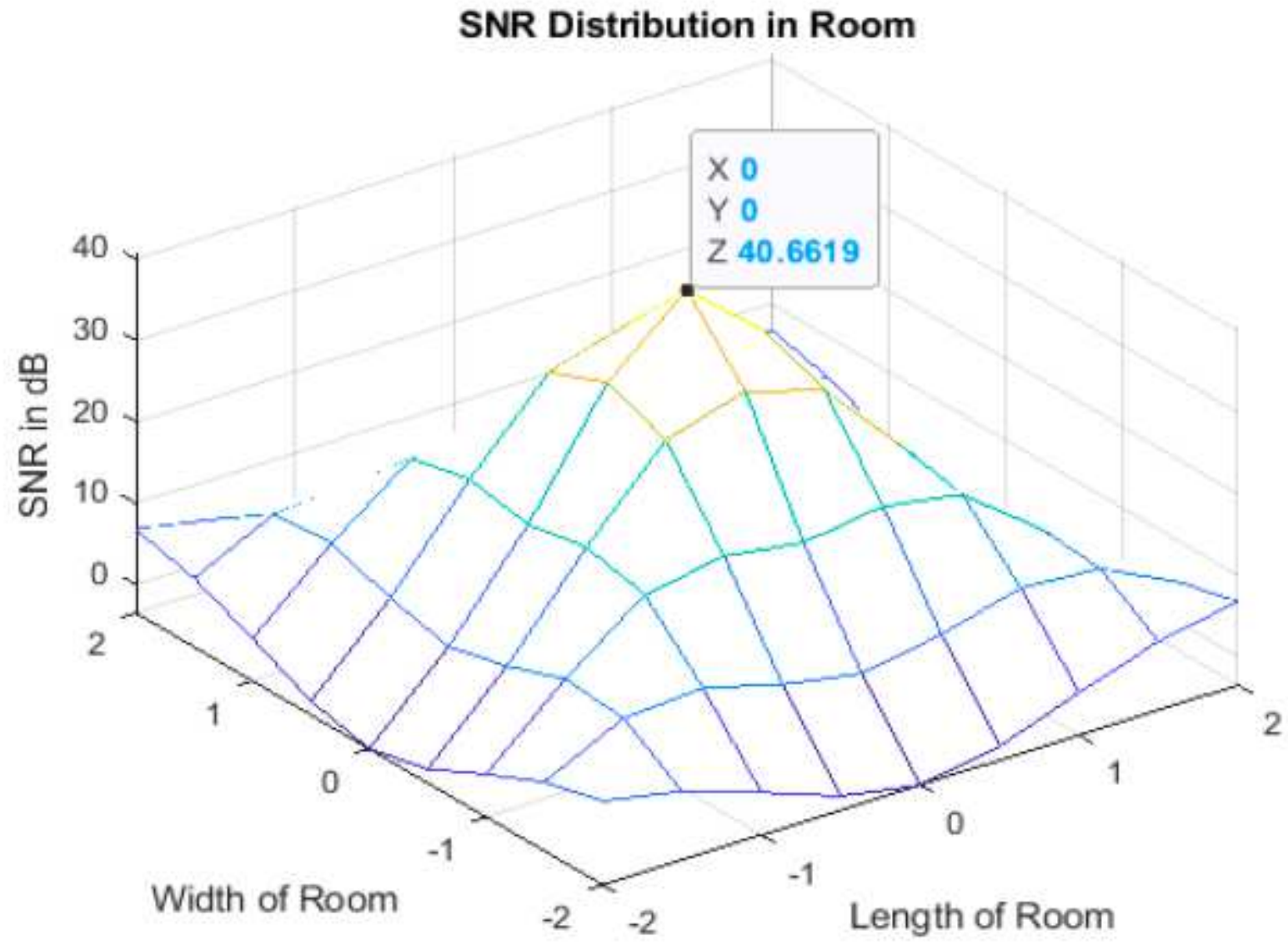

Figure 5

SNR analysis of proposed system for $16 \mathrm{X} 16$ elements 


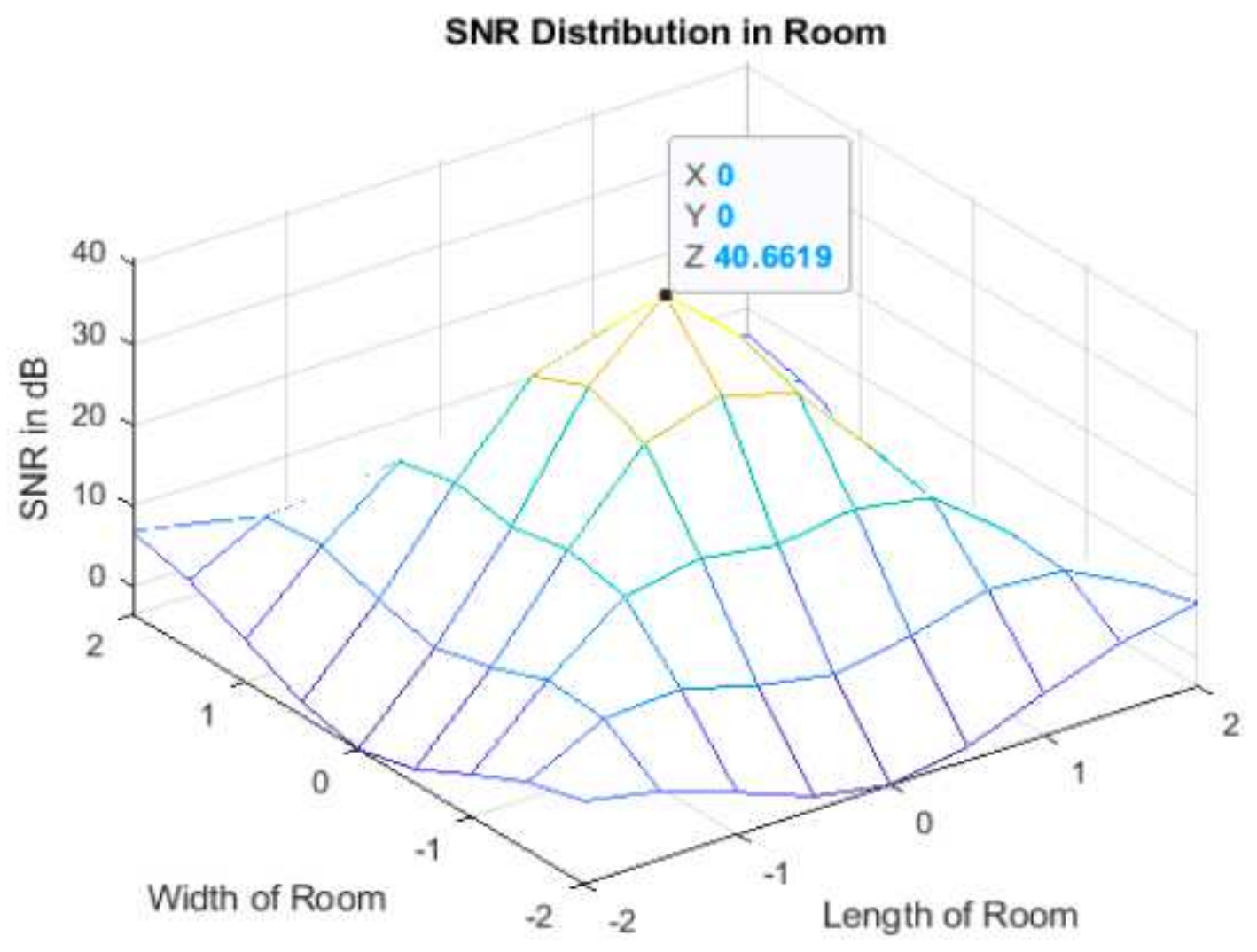

Figure 6

SNR analysis of existing system for $32 \times 32$ elements 


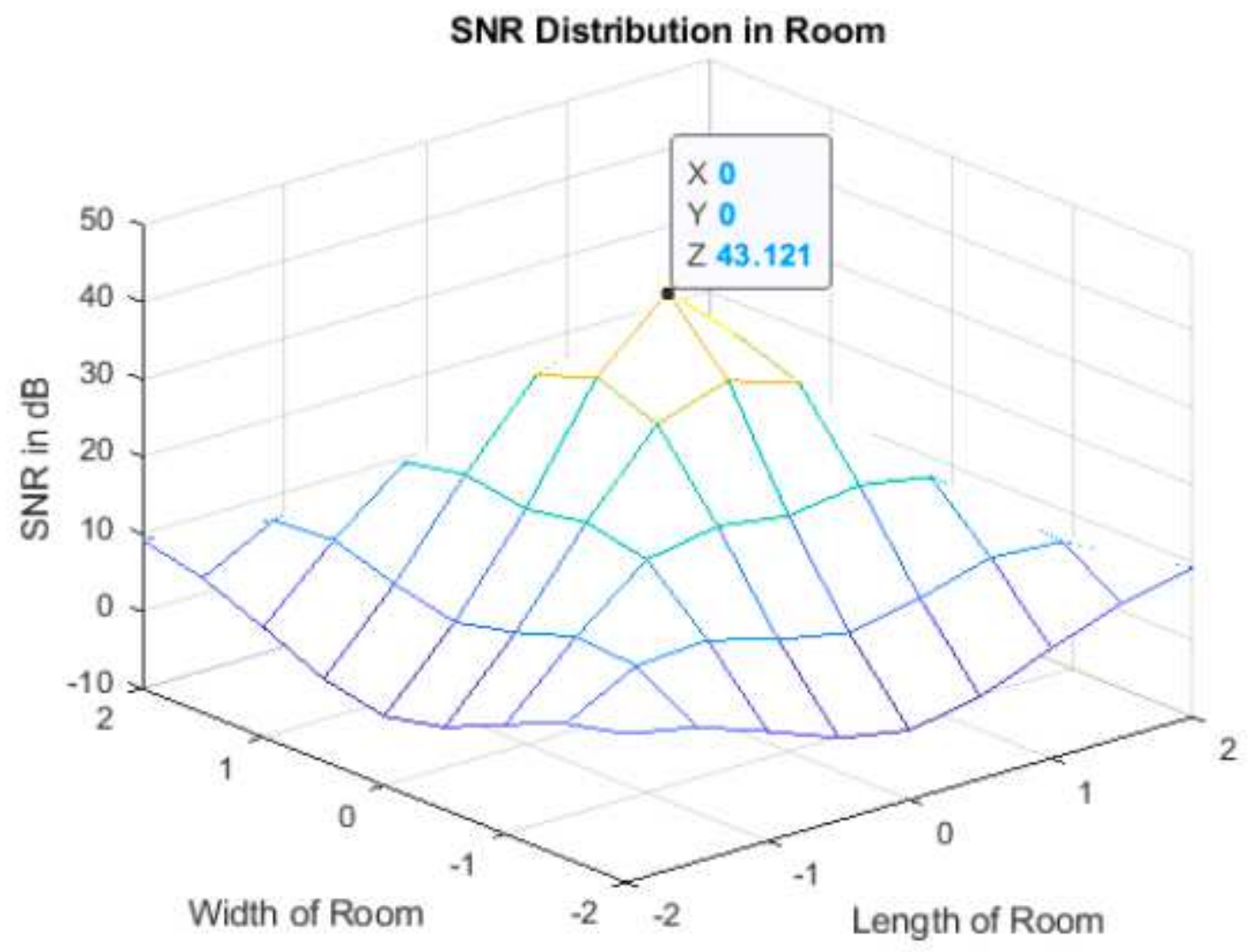

Figure 7

SNR analysis of proposed system for $32 \times 32$ elements 


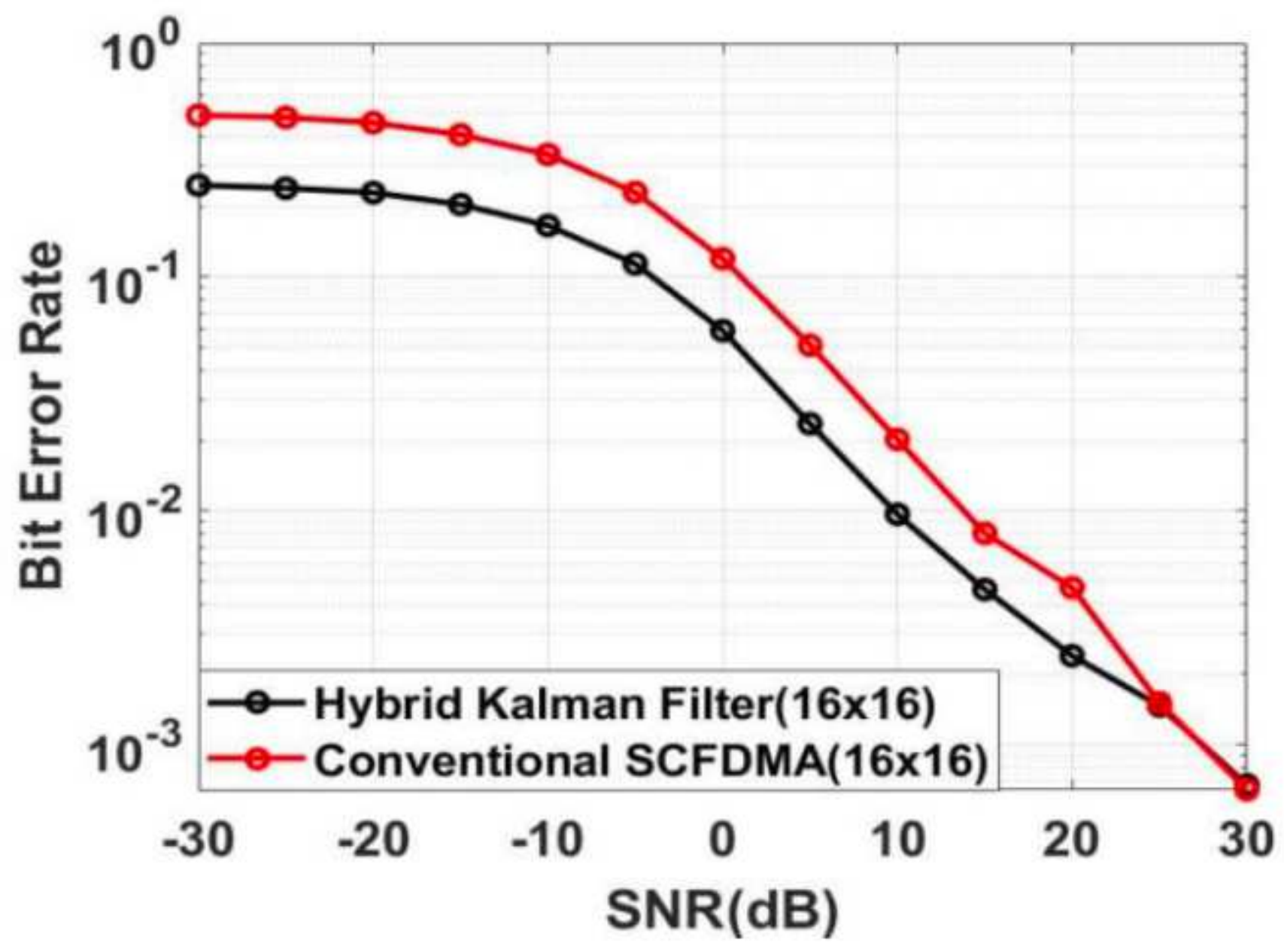

Figure 8

BER vs SNR for hybrid Kalman Filter and SCFDMA for 16X16 elements 


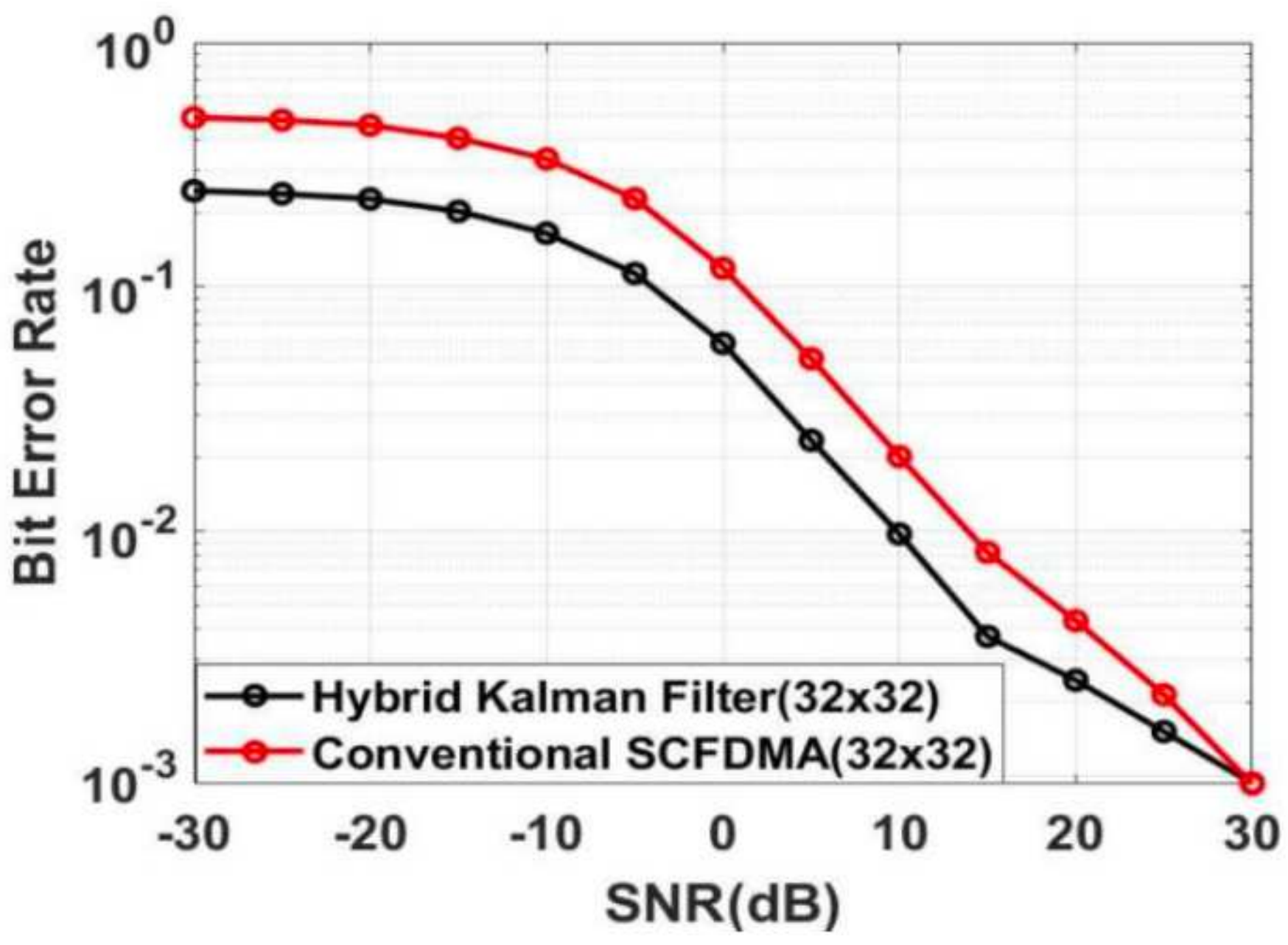

Figure 9

BER vs SNR for hybrid Kalman Filter and SCFDMA for 32X32 elements 